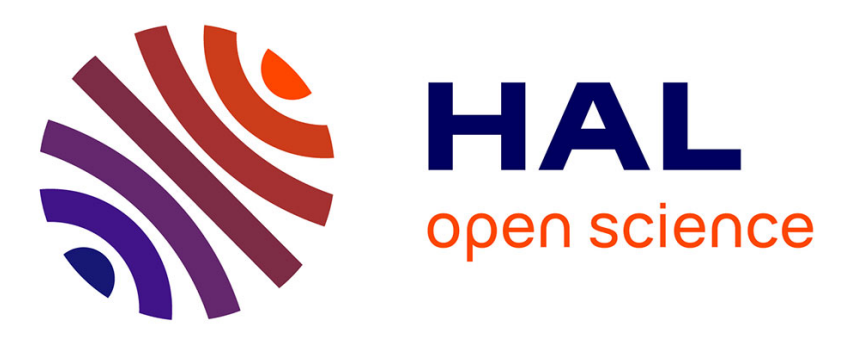

\title{
Beneficial impact of wave-breaking for coherent continuum formation in normally dispersive nonlinear fibers
}

Christophe Finot, Bertrand Kibler, Lionel Provost, S. Wabnitz

\section{To cite this version:}

Christophe Finot, Bertrand Kibler, Lionel Provost, S. Wabnitz. Beneficial impact of wave-breaking for coherent continuum formation in normally dispersive nonlinear fibers. Journal of the Optical Society of America B, 2008, 25 (11), pp.1938-1948. 10.1364/JOSAB.25.001938 . hal-00408594

\section{HAL Id: hal-00408594 \\ https://hal.science/hal-00408594}

Submitted on 17 Apr 2010

HAL is a multi-disciplinary open access archive for the deposit and dissemination of scientific research documents, whether they are published or not. The documents may come from teaching and research institutions in France or abroad, or from public or private research centers.
L'archive ouverte pluridisciplinaire HAL, est destinée au dépôt et à la diffusion de documents scientifiques de niveau recherche, publiés ou non, émanant des établissements d'enseignement et de recherche français ou étrangers, des laboratoires publics ou privés. 


\title{
Beneficial impact of wave-breaking
}

\section{for coherent continuum formation}

\section{in normally dispersive nonlinear fibers}

\author{
Christophe FINOT, ${ }^{1 *}$ Bertrand KIBLER, ${ }^{1}$ Lionel PROVOST, ${ }^{2}$ and Stefan WABNITZ ${ }^{1}$ \\ ${ }^{1}$ Institut Carnot de Bourgogne (ICB), UMR-CNRS 5209, Université de Bourgogne, \\ 9 Avenue Alain Savary, 21078 Dijon, France \\ ${ }^{2}$ Optoelectronics Research Centre, University of Southampton, SO17 1BJ, United-Kingdom \\ *Corresponding author: christophe.finot@u-bourgogne.fr
}

We study the evolution of a pulse propagating in a normally dispersive fiber in presence of Kerr nonlinearity. We review the temporal and spectral impact of optical wave-breaking in the development of a continuum. Impact of linear losses or gain is also investigated. (C) 2008 Optical Society of America

OCIS codes: $060.4370,060.7140,060.5530$. 


\section{Introduction}

Continuum light sources have generated a great deal of attention over the past decade: examples of spectral broadenings as large as a decade have been reported using optical fibers as nonlinear and dispersive propagation medium [1]. The areas of application of supercontinuum (SC) sources are widespread, ranging from biology [2] to optical metrology [3]. Optical telecommunications have also benefited from the development of continuum light source technologies [4], for example in the context of wavelength-division multiplexed systems where a high number of optical channels can be generated through the spectral slicing of spectrally broadened picosecond pulses [5-8].

As it is well known, the physical phenomena underlying the spectral evolution of a short optical pulse in a fiber will significantly differ, depending on the sign of the fiber group-velocity dispersion (GVD). On the one hand, anomalous dispersion generates (through soliton compression and subsequent fission) the broadest spectra reported to-date [7]. In this case, however, spectral broadening is accompanied by relatively high timing and amplitude jitter of the pulses [9]. On the other hand, although optical pulse evolution in a normally dispersive fiber leads to relatively narrower spectra, in this case a reduced spectral ripple and initial pulse jitter influence are both observed $[5,6,8]$. Hence frequency broadening using a normally dispersive fiber appears as an attractive choice whenever a moderate amount of broadening alongside with good spectral and temporal stability of the output pulses are targeted [10], for applications such as optical regeneration [11-13] or pulse temporal compression [5, 14, 15].

Experimentally, a wide range of normally dispersive fibers have been successfully tested for SC generation: for example, dispersion shifted fibers [8], highly nonlinear silica fibers [5, 10], photonic crystal fibers [6], and dispersion decreasing normally dispersive fibers [16]. New 
specialty glasses exhibiting very high values of normal dispersion have also been investigated [13] demonstrating possible benefits of such glasses to improve overall power budget and device footprint. Since in all of the above practical implementations the dispersion, the nonlinear properties as well as the fiber losses may vary over different orders of magnitude, it would be of high interest if one could develop some general, and possibly analytical, design guidelines for the understanding and prediction of the temporal and spectral properties of the generated pulses.

In the present article, we intend to provide an answer to the above demand by reconsidering through a unified and, as far as we know, original approach the old problem of pulse evolution in a normally dispersive optical fiber. It is well known that upon propagation in the normal GVD regime a short pulse may experience a strong distortion of its temporal shape (e.g., a flattening of its top) [17]. One of the critical phenomena affecting short pulse dynamics in the normal GVD regime is known as "optical Wave-Breaking" (WB), which results from the temporal overlap of the highly chirped central part of the pulse with its un-chirped wings [18]. This phenomenon has long been seen as a deleterious effect which has in general to be avoided in practice, with the help of parabolic pulse shaping for example [19]. Quite surprisingly, we will point out in this work that the presence of WB may even turn out to be beneficial in a variety of applications.

Our paper will be organized as follows. At first we shall describe our model equations that are based on the standard nonlinear Schrödinger Equation (NLSE). Next we will review the different steps that are involved in the development of a continuum. We will then provide an original interpretation of how wave-breaking may affect the evolution (both in the temporal and spectral domains) of the continuum in the normal GVD regime. Our fine understanding of the pulse dynamics will lead us to propose simple design rules that enable one to optimize the 
continuum development. Indeed, in a next section we shall describe the results of our experimental frequency broadening studies that allowed us to validate the above discussed analytical trends. Finally, in the last section of our work we will extend our conclusions to the case where losses or gain contribution are included.

\section{Numerical model}

The longitudinal evolution in an optical fiber of the complex electric field envelope $\psi(z, T)$ can be modeled in terms of the generalized NLSE $[1,20]$ :

$$
i \frac{\partial \psi}{\partial z}=-i \frac{\alpha}{2} \psi-\sum_{k \geq 2} \frac{i^{k} \beta_{k}}{k !} \frac{\partial^{k} \psi}{\partial T^{k}}-\gamma\left(1+\frac{i}{\omega_{0}} \frac{\partial}{\partial T}\right) \times \psi \int_{-\infty}^{T} R\left(T^{\prime}\right)\left|\psi\left(T-T^{\prime}\right)\right|^{2} d T^{\prime},
$$

where $\beta_{\mathrm{k}}$ is the $\mathrm{k}^{\text {th }}$-order dispersion coefficient, and $\gamma$ is the nonlinear coefficient. $\alpha>0 \quad(\alpha<0)$ is the fiber loss (distributed gain) coefficient. $R(T)=\left(1-f_{R}\right) \delta_{K}(T)+f_{R} h_{R}(T)$ includes instantaneous $\delta_{K}(T)$ and delayed Raman $h_{R}(T)$ contributions with the fractional Raman contribution $f_{R} \cdot 1 / \omega_{0} \partial / \partial T$ is the self-steepening term. $T$ is the time coordinate in a frame of reference that co-propagates with the pulse, and $z$ the propagation distance.

In this work, we aim to describe continuum development (or the first steps of supercontinuum dynamics), i.e. when the pulse spectral width is on the order of a few THz. For such a spectral width, self-steepening has not found as a determining effect. Moreover, assuming a dispersion flattened fiber, we can neglect higher order dispersion terms (third order dispersion will induce as a first consequence to some spectral asymmetry [10, 21]). In normally dispersive fibers, intrapulse Raman scattering has a reduced impact and as long as the fiber length or initial power is below stimulated Raman threshold, Raman term can also be neglected. 
In this context, Eq. (1) reduces to the simplified NLSE expression [20] :

$$
i \frac{\partial \psi}{\partial z}=\frac{\beta_{2}}{2} \frac{\partial^{2} \psi}{\partial T^{2}}-\gamma|\psi|^{2} \psi-i \frac{\alpha}{2} \psi
$$

In order to make our results applicable to a wide range of experimental situations, it is beneficial to transform Eq. (2) into the following normalized form [20]

$$
i \frac{\partial u}{\partial \xi}=\frac{1}{2} \frac{\partial^{2} u}{\partial \tau^{2}}-|u|^{2} u-i \frac{\delta}{2} u
$$

where $u, \tau$ and $\xi$ are the normalized parameters defined as

$$
u(\xi, \tau)=N \quad U, \quad U(\xi, \tau)=\frac{\psi}{\sqrt{P_{C}}}, \quad \tau=\frac{T}{T_{0}}, \quad \delta=\alpha L_{D}, \quad \xi=\frac{z}{L_{D}}
$$

Here $T_{0}$ and $P_{C}$ represent the characteristic temporal width and peak power of the initial pulse, respectively. $L_{D}, L_{N L}$ and $N$ are the dispersion length, the nonlinear length and the "soliton" order, and are defined as follows

$$
L_{D}=\frac{T_{0}^{2}}{\beta_{2}}, \quad L_{N L}=\frac{1}{\gamma P_{C}}, \quad N=\sqrt{\frac{L_{D}}{L_{N L}}} .
$$

In order to quantitatively characterize the evolution along the fiber of the field $u$, various approximate approaches have been recently developed. Ruehl et al. have used the kurtosis parameter [22]. Jirauschek et al. have based their analysis on the use of an ansazt [23], whereas the analysis of Burgoyne et al. or Rosenberg et al. employed the method of moments [24, 25]. In the present work, we based our investigation on extensive numerical integrations of Eq. (3) by means of the standard split-step Fourier algorithm [20]. To grasp a global portrait of the pulse 
propagation, we have selected, computed and displayed some characteristic parameters that may conveniently describe the temporal and spectral properties of a propagated pulse. Figure 1 illustrates some of these parameters for a typical output pulse after propagation down the normally dispersive fiber.

The first key parameters are $\tau_{-3}$, which represents the temporal broadening of a pulse (defined as the evolution of the Full temporal Width at Half-Maximum (FWHM) of the intensity profile), and the pulse peak power reduction factor (defined as the ratio between the output and the input peak power). In order to characterize the slope of the pulse wings, we have estimated the $S$ parameter, which we have defined as follows

$$
S=-2 \frac{u_{-1}^{2}-u_{-10}^{2}}{\tau_{-1}-\tau_{-10}} \frac{\tau_{-3}}{u_{0}^{2}} \simeq 1.39 \frac{\tau_{-3}}{\tau_{-10}-\tau_{-1}}
$$

In the above expressions, $u_{-x}^{2}$ (and $\tau_{-x}$ ) are the pulse power (and its associated temporal width) at $-x \mathrm{~dB}$ with respect to the peak power value at $\tau=0$.

We are also interested in characterizing the shape (in particular, the flatness) of the top of a pulse. To this aim, we develop the pulse intensity profile in terms of a Taylor's expansion centered at the pulse's mid-point (i.e., for $\tau=0$ )

$$
\left.\left.\left.u^{2}(\tau)=u_{0}^{2}+\frac{\partial u^{2}}{\partial \tau}\right)_{\tau=0} \tau+\frac{\partial^{2} u^{2}}{\partial \tau^{2}}\right)_{\tau=0} \frac{\tau^{2}}{2}=u_{0}^{2}+\frac{\partial^{2} u^{2}}{\partial \tau^{2}}\right)_{\tau=0} \frac{\tau^{2}}{2}
$$

In order to estimate the flatness of the pulse shape, we may introduce the following dimensionless quantity

$$
\left.F=\mid \frac{\partial^{2} u^{2}}{\partial \tau^{2}}\right)_{\tau=0} \mid \frac{\tau_{-3}^{2}}{u_{0}^{2}}
$$


To characterize the pulse evolution in the frequency domain, we have estimated the broadening of its spectral intensity profile. Since we would like to differentiate the evolution of the central part of the spectrum from the evolution of its wings, we have computed both the $-3 \mathrm{~dB}$ and the $-20 \mathrm{~dB}$ spectral bandwidths. We have also drawn a special care to measuring the spectral ripple of the output pulse by computing corresponding ripple in the particular spectral range that is delimitated by the two observed outermost peaks. Finally, the parameter that we have used to evaluate the frequency confinement of the pulse energy was defined by the ratio between the energy comprised in the $-3 \mathrm{~dB}$ range and the total pulse energy, respectively.

\section{Wave-breaking}

In order to provide a simple physical interpretation of pulse propagation in a normally dispersive fiber, let us review in this section the development of the so-called optical wave-breaking phenomenon. Indeed, it has been known for a long time that the combination of normal dispersion and nonlinearity may lead to dramatic pulse distortions [18, 26]. For the sake of simplicity, we shall neglect in this section the impact of either loss or gain ( $\delta=0$ ). In order to better visualize the effects of WB, we highlight the benefit of the pulse spectrogram that allows a convenient derivation of both temporal and frequency evolutions from the same data. This kind of representation can be experimentally recorded, by means of a streak spectrometer for example [27] or a XFROG device [28]. Other time/frequency representations are possible, such as the Wigner representation [29].

In Figure 2 we have plotted the evolution of a pulse with an initial un-chirped sech shape, i.e. $u(0, \tau)=N \operatorname{sech}(\tau)$, and with $N=40$. The initial pulse, which is by definition transformlimited, is shown in Fig. 2(a). In the first stages of its propagation, the major physical effect 
acting on the pulse of Fig.2 is Self-Phase Modulation (SPM) [30], which introduces a chirp (or instantaneous frequency shift) $C_{n l}$ that reads as

$$
C_{n l}(\xi, \tau)=-\xi \frac{\partial}{\partial \tau}\left(u^{2}(0, \tau)\right)
$$

In the spectral domain, the SPM-induced chirp (8) may lead to a significant frequency broadening of an optical pulse. Since the resulting instantaneous frequencies do not spread monotonically across the pulse temporal profile, chirping introduces spectral oscillations at the pulse's edges, as shown in Fig. 2(b). Note that in the initial stage of pulse propagation of Fig.2(b), the interaction of SPM with GVD also changes the initial hyperbolic secant pulse temporal profile into a parabolic one [26,31]. Under the action of normal GVD, the intermediate pulse sections of Fig. 2(b) where the instantaneous frequency attains the maximum or minimum values propagate at different speeds than their respective outer pulse tails. For example, the leading intermediate section of the pulse (where the instantaneous frequency attains the largest down-shift) propagates faster than the leading pulse tail. This eventually results in the faster intermediate section taking over the slower preceding tail, as it can be seen on Fig. 2(c). In the temporal domain, an overlapping of two pulse components with different instantaneous frequencies (also known as WB) entails the appearance of a sinusoidal beating between those frequencies [32]. Such beating in turn creates new frequencies through four-wave mixing (FWM) in the fiber, as it is clearly visible at the edges of the spectrum which is shown in Fig. 2(c). We may also point out in Fig. 2(c) that the occurrence of wave-breaking is accompanied by an enhancement of the flatness of the central part of the spectrum. Such spectral region is associated with a nearly linear distribution of the instantaneous frequency across the pulse. In other words, if the objective is to achieve a pulse with high spectral flatness, it proves convenient 
to propagate the input pulse until WB occurs. Note that, since the WB distance can be minimized by increasing the absolute value of the group velocity dispersion, it can be beneficial using a fiber with a relatively high dispersion value [13]. It can be further noticed in Fig. 2(c) that the central part of the pulse spectrum (i.e., for $v=0$ ) results from separate contributions at three different points in time (i.e at $\tau=-2.5,0$ and 2.5). As a consequence, enhanced intensity oscillations near the center of the pulse spectrum are observed.

Finally, Figure 2(d) shows the evolution of the pulse after WB has occurred: the central part of the spectrum does not broaden anymore as the distance grows larger. To the contrary, the pulse has undergone a significant broadening in the temporal domain. Moreover, the central section of the pulse is progressively flattened in both the temporal and in the frequency domain. Note also in Fig. 2(d) that, owing to the normal GVD of the fiber, the newly generated frequencies in the wings of the spectrum have completely overtaken (or have left behind) the leading (trailing) edges of the initial pulse. Therefore the overlap among pulse components with different instantaneous frequencies and the associated FWM no longer occurs.

\section{Temporal evolution}

In this section we shall focus in a more systematic manner to the description of the temporal evolution of pulses upon their propagation in a normally dispersive fiber. To illustrate our description, we will consider the case of an initial Gaussian pulse $u(0, \tau)=N \exp \left(-\tau^{2} / 2\right)$.

Let us first discuss the evolution of the FWHM temporal width the Gaussian pulses as a function of the normalized propagation distance $\xi$ and of the initial power $N$. The corresponding results are shown by means of contour plots in Fig. 3(a): as it can be seen, at relatively low initial powers or for short propagation distances the temporal pulse broadening is only moderate. Fig. 3(a) also shows that the higher is the nonlinearity, the faster is the temporal pulse 
broadening. This results from the strong interaction between nonlinearity and dispersion. In order to better understand such interaction, it proves very helpful to plot the analytical condition for the onset of WB as it was derived by Anderson et al [26]. Such condition relates the distance $\xi_{w b}$, where WB is first observed, to the soliton order $N$ as

$$
\xi_{w b}=\frac{1}{\sqrt{4 \exp (-3 / 2) N^{2}-1}}
$$

which reduces, in the limit of high $\mathrm{N}$, to the simpler expression

$$
\xi_{w b} N \simeq \frac{\exp (3 / 4)}{2} \simeq \text { constant }
$$

The relationship (9) is plotted in Fig. 3(a) as a white solid line: as it can be seen, Eq.(9) describes the border between two different regions in the $(\xi, N)$ plane. In the region situated below the white curve pulse broadening remains moderate, indicating that in this region SPM is the dominant physical mechanism influencing pulse evolution. Whereas in the region situated above the white curve the interplay of SPM and GVD leads to an accelerated temporal broadening. From a systems point of view, in the case of telecommunications signals with a moderate duty cycle (typically $25 \%$ or $33 \%$ ), it is crucial to control and limit the temporal broadening of a signal pulse in order to avoid inter-symbol distortions arising from pulse-to-pulse interactions [33]. Such distortions could be especially deleterious in applications of nonlinear optical fibers to optical regeneration devices [12].

Next we plotted in Fig. 3(b) the evolution of the peak power of the pulse in the $(\xi, N)$ plane. Since we consider here the propagation of constant energy pulses, pulse peak power and temporal duration are necessarily linked with each other : pulse broadening in time is thus 
accompanied by a drop of the peak power. Indeed, Fig. 3(b) shows that indeed for points lying at the threshold defined by Eq. (10), the input peak power has dropped by a factor of 2 .

In order to visualize the evolution of pulse shape, we have computed the $S$ and $F$ parameters as we have defined them through Eq. (6) and (8), respectively. The corresponding results are plotted in Fig. 4(a), and clearly demonstrate that WB (white line) occurs at the edge of a significant a pulse steepening region. This observation is in agreement with the results outlined by Anderson et al. [26], who have used a similar parameter in order to detect the wave-breaking stage. Fig. 4(b) highlights that above the WB threshold the concavity of the central region of the pulse is significantly decreased, which is indicative of a strong flattening of the pulse top, as it was also observed in Fig. 2(d).

Finally, it proves interesting to monitor the evolution of the chirp coefficient $\mathrm{C}_{n l}$ of Eq.(8), which is numerically obtained by computing the slope of the pulse chirp (i.e., for $\tau=0$ ). The corresponding results are illustrated in Fig. 4(c). We can see that the temporal chirp is maximal in the high-N region, and before the WB has occurred. Indeed, before the onset of WB, the pulse has not been temporally broadened yet. As a result, all of the spectral components of the pulse remain packed in time near the pulse top at $\tau=0$, which results in a large temporal rate of variation of the pulse intensity, hence a large chirp coefficient. After the occurrence of WB, the pulse significantly broadens in time, so that its instantaneous frequencies are dispersed, the pulse top flattens and chirp coefficient decreases.

\section{Spectral evolution}

Let us concentrate our attention in this section on the spectral evolution of the pulse when SPM and normal GVD are experienced. As we shall see, the occurrence of the wave-breaking 
phenomenon has a major role in determining the properties of the spectral reshaping of a pulse. We will study at first the evolution in the $(\xi, N)$ plane of the $-3 \mathrm{~dB}$ spectral width of the pulse (see Fig. 5(a)). As it can be seen, the pulse progressively broadens its spectrum until a maximum broadening factor is reached. Upon further propagation, a saturation of the spectral broadening occurs, until the spectral width eventually decreases with distance. In Fig. 5, we have indicated with a white solid line the WB distance $\xi_{\mathrm{WB}}$ of Eqs.(9-10), alongside with the empirical formula that was proposed by Taccheo et al. [34] for describing the distance where maximum nonlinear spectral expansion is observed for a Gaussian pulse

$$
\xi N=2.1=\text { constant }
$$

By comparison with the numerical results of Fig.(5), it turns out that Eq. (12) describes the spectral broadening saturation stage with better accuracy than Eqs.(9-10). The connection between Eq.(11) and the WB effect described in Eqs. (11), is nevertheless interesting since it permits to physically justify to some extend the empirical $1 / N$ scaling from Eq. (11). As a matter of fact, as we will better clarify below, the observed saturation in the pulse spectral broadening is closely linked to the presence of WB. We may also notice in Fig. 5(a) the presence of a second high-spectral broadening region where $N \gg 10$ and $\xi>0.1$. The corresponding distances are such that the WB-generated spectral wings grow higher than the $-3 \mathrm{~dB}$ boundary. Similar behavior will also be observed for initial sech pulse (Fig. 6).

Let us now try to provide a physical interpretation of the observed spectral saturation. By using Eqs. (7)-(7), we may approximate the SPM-induced chirp $\delta C_{n l}$ at the pulse center after the propagation distance $\delta \xi(E q .(9))$ as 


$$
\delta C_{n l}=\delta \xi \quad F \quad \frac{u_{0}^{2}}{\tau_{-3}^{2}} \tau
$$

The above equation shows that the chirp is proportional to the peak-power $u_{0}^{2}$, to the flatness $F$ of the pulse, and it is inversely proportional to the temporal pulse width $\tau_{-3}^{2}$. Therefore, as we have outlined in the previous section, after the occurrence of WB one obtains a decrease of the peak power of the pulse (Fig. 3(b)), a broadening of its time width (Fig. 3(a), and an increase in its flatness $($ Fig. $4(b))$. As a result, the additional self-phase modulation $\delta C_{n l}$ that is introduced by the central part of the pulse will tend to decrease.

In order to infer what the maximum spectral broadening actually is, we may follow the simple qualitative reasoning. The chirp that is induced by SPM only for a Gaussian pulse of amplitude $N$ and after a propagation length $\xi$ is (given that $N \gg 1$ ) reads as

$$
C_{n l}^{\text {Gauss }}(\xi, \tau)=2 \xi N^{2} \tau \exp \left(-\tau^{2}\right)
$$

so that we may qualitatively evaluate the maximal frequency shift that is induced by self-phase modulation as

$$
\Delta C_{n l}^{\text {Gauss }}(\xi)=\max \left(C_{n l}^{\text {Gauss }}(\xi, \tau)\right)-\min \left(C_{n l}^{\text {Gauss }}(\xi, \tau)\right)=\frac{4}{\sqrt{2}} \xi N^{2} e^{-\frac{1}{2}}
$$

If we consider that WB occurs after the distance $\xi_{w b}$ (see Eq. (11)), we obtain the following expression for the spectral broadening (defined as the ratio between the maximum FWHM spectral width and the initial FWHM spectral width of the pulse) : 


$$
\exp \left(\frac{1}{4}\right) \frac{\sqrt{2 \ln (2)}}{0.44 \pi} \quad N \simeq 1.1 N
$$

As it can be seen in Fig. 5(b), the predictions from Eq. (16) are in good agreement with the numerical results, and are also in excellent agreement with the empirical linear growth of the spectral broadening (i.e., proportional to $1.1 \mathrm{~N}$ ) which was proposed in ref. [34].

Let us focus now our attention on the evolution of the $-20 \mathrm{~dB}$ spectral width that is presented in Fig. 5(c). We may notice that in this case the spectral broadening increases at first monotonically with distance. After a maximum broadening is reached, the spectral width tends to saturate, albeit at longer distances than the FWHM spectral width. By computing the ratio between the FWHM spectral width and the $-20 \mathrm{~dB}$ spectral width, we may better understand the correlation between the evolutions of the two quantities. This ratio is shown in Fig. 5(d), which shows that, after the WB occurs, the two widths tend to a specific value which is close to two. Such ratio of spectral widths is directly linked with the nonlinear process that originates the build-up of the spectral wings of the pulse. Indeed, as we have seen on Fig. 2(c), the pulse wings are generated through the FWM interaction occurring at $\tau= \pm 2.5$ between the peak frequencies of the pulse ( with an instantaneous frequency $v= \pm 5$ ) which act as a pump, and the pulse tails at $v=0$ which act as a seed. Note that, given the sharpness the spectrum edges, the peak frequencies of the pulse are close to \pm the half FWHM spectral width. As a result, the frequency of the resulting FWM-generated photons is equal to \pm the pulse FWHM spectral width, which leads to a $-20 \mathrm{~dB}$ spectral width that is nearly twice as large as the original FWHM bandwidth. 
For practical applications of SC pulses, it is also interesting to consider the evolution with distance of the energy which is stored in the central part on the pulse spectrum. The impact of WB on the pulse spectral energy distribution is readily apparent on the contour plot of Fig. 6(a). Below the threshold, most of the pulse energy is indeed contained within the $-3 \mathrm{~dB}$ bandwidth of the pulse spectrum. After the onset of WB, a significant energy transfer into the pulse spectral wings is observed [18]. If a main objective of the generated SC light is to make sure that the pulse energy is well confined within the central portion of its spectrum, then it would be beneficial to operate below the WB limit distance. As discussed above, the pulse spectral energy re-distribution from its center towards its wings occurs via FWM, where each extreme frequency of the main spectral region of pulse acts as a pump. As those frequencies get depleted, one observes a narrowing of the $-3 \mathrm{~dB}$ spectral bandwidth, as already pointed out with reference to Fig. 5(a).

Finally, let us study the evolution of the spectral ripple which is observed in the central region of the pulse spectrum. By examining the simulation results that are shown in the contour plot of Fig. 6(b), we may easily distinguish two distinct regions. Below the WB threshold, the pulse spectrum exhibits a relatively high-amplitude ripple, as it was also previously outlined in section 3. Once the WB threshold is overcome, the spectral ripple is dramatically decreased and, as a result, the spectrum is basically flat. Therefore, in situations where the practical target involves slicing the pulse spectrum for pulse spectral shaping [35] or for implementing a multiwavelength source, it may be beneficial to operate beyond the WB distance.

So far in our analysis we focused our attention on the evolution of a Gaussian initial pulse. In Refs. $[26,31]$ it was pointed out that transient states towards WB were dependent on 
the initial pulse shape. This is still true for the evolutions described here. In order to illustrate this point, let us consider the evolution of two other input pulse shapes, i.e., an hyperbolic secant pulse and a parabolic pulse (which is defined as $|u(\tau, 0)|^{2}=N^{2}\left(1-\tau^{2}\right)$ ). We show the fraction of spectral energy which is contained within the $-3 \mathrm{~dB}$ bandwidth as a function of $\mathrm{N}$ and $\xi$ in Figs.7(a) and 7(b) for the case of an input hyperbolic secant (or sech) or parabolic pulse, respectively. By comparing the results of Fig.6(a) and Fig.7(a), one may observe that the dynamics of a sech pulse and of a Gaussian pulse are qualitatively similar. Therefore, the treatment of Ref. [26] provides the following analytical prediction for the distance $\xi_{w b S}$ at which WB is observed with input sech pulses and for high $N$ 's :

$$
\xi_{w b S}=\sqrt{\frac{3}{2}} \frac{1}{\sqrt{N^{2}+1}} \simeq \sqrt{\frac{3}{2}} \frac{1}{N}
$$

By following the same reasoning which led us to qualitatively estimate the spectral broadening of Gaussian pulses, we may estimate the maximum spectral broadening for sech pulses as

$$
\frac{2^{\frac{3}{2}}}{3 \pi} \frac{2 \ln (1+\sqrt{2})}{0.31} N
$$

The above result is plotted as a grey curve on Fig. $5(\mathrm{~b})$. The relative error between the analytical estimation (17) and the corresponding numerical results (grey circles) is within $12.5 \%$, which is much larger than the small error that is obtained with Gaussian input pulses. Nevertheless, our analytical results in Fig.5(b) confirm the numerical observation that by using an initial sech pulse one obtains larger spectral broadenings than with a Gaussian pulse, as it was already empirically pointed out in Ref. [34]. Furthermore, our present treatment permits to physically justify the observed linear scaling of the spectral broadening factor as a function of $N$. 
On the other hand, Fig. 7(b) which was obtained for the case of a parabolic initial pulse shows that in this case the spectral broadening undergoes a rather different evolution. Quite remarkably, for a parabolic input pulse the spectral power density does not decrease as both $\mathrm{N}$ and $\xi$ grow larger. Indeed, Fig. 7(b) shows that a relatively high spectral density is maintained at a high propagation distances. This result is consistent with the well-known ability of a parabolic pulse to resist to WB $[19,36]$, as well as to avoid a transfer of energy from the central part of the pulse spectrum into its spectral wings through FWM. As a matter of fact, the significant robustness of parabolic pulses with respect to WB has been used for optimized spectral slicing applications, where the parabolic intensity profile was obtained by linear pulse shaping with a superstructure Bragg grating [5].

\section{Experiments}

The above discussed numerical experiments provide us with general guidelines for the explanation of the observed temporal and spectral dynamics of optical pulses that propagate in a normally dispersive and nonlinear fiber. In particular, we could point out that WB sets a clear borderline in both the quantitative and qualitative properties of the pulse evolution in both time and frequency domains. Based on our numerical studies, we have carried out a systematic experimental analysis with the purpose to verify the validity of the theoretical general trends that were discussed in the previous sections.

Our experimental set up is sketched in Fig. 8. Initial 4 ps temporal width sech pulses at $1552.5 \mathrm{~nm}$ were delivered by a picosecond erbium-doped fiber laser operating at the repetition 
rate of $22 \mathrm{MHz}$. The average power of the pulse train from the laser was increased by means of an erbium-doped fiber amplifier (EDFA). Amplified spontaneous emission noise was removed by means of an optical bandpass filter $(O B P F)$, whereas an optical variable attenuator $(O V A)$ was used to control the peak power that was launched into the optical fiber. Let us note that pulse amplification by the EDFA was accompanied by SPM, which led to a slight temporal compression (to a resulting temporal FWHM of $3.8 \mathrm{ps}$ ). Moreover, the associated SPM-induced chirp and spectral broadening led to launching non-transform-limited pulses into the test optical fiber.

The fiber that was used in our experiments was a non-zero dispersion shifted fiber (NZ$D S F$, TrueWave fiber by OFS) with the second-order dispersion $\beta_{2}=3.2 \times 10^{-3} \mathrm{ps}^{2} . \mathrm{m}^{-1}$, the nonlinear coefficient $\gamma=1.7 \mathrm{~W}^{-1} \cdot \mathrm{km}^{-1}$, and linear losses $\alpha=0.2 \mathrm{~dB} \cdot \mathrm{km}^{-1}$ (which led to a negligible influence of fiber loss in our experiments). We used an initial fiber length $L=609 \mathrm{~m}$ (corresponding to $\xi=0.42$ ); next, in order to monitor the output pulses for a set of different propagation distances, we have progressively reduced the fiber length by removing $29 \mathrm{~m}$ at each cut. For each specific value of fiber length, we recorded the output spectra for a set of input powers ranking from $0.4 \mathrm{~W}$ to $150 \mathrm{~W}$, i.e., the $N$ values varied from 1 to 20 .

These systematic measurements enabled us to draw maps in the $N-\xi$ plane for illustrating the different spectral properties of the output pulses, as shown in Fig. 9. Among the resulting set of 420 spectra, we removed the few experimental points where the spectral evolution was affected by the generation of a Raman Stokes wave [37]. We have also experimentally checked that the third-order dispersion of the fiber $\left(\beta_{3}=2 \times 10^{-5} \mathrm{ps}^{3} \cdot \mathrm{m}^{-1}\right)$ was not responsible for significant modifications of the pulse spectrum, i.e., only little spectral asymmetry was observed in our measurements $[10,21]$. More importantly, we experimentally observed that the symmetry 
of the initial pulse was of crucial importance in order to observe a symmetric broadening of the output spectrum.

The experimental results shown in Fig. 9 demonstrate a good qualitative agreement with the theoretical trends that were outlined in section 5 (compare Fig. 9 (a-c) with Fig. 5(a,c) and Fig. 6(b), respectively). Indeed, the experiments of Fig. 9(a) confirm the predicted saturation with fiber distance of the $-3 \mathrm{~dB}$ spectral width of the pulse. Fig. 9(b) shows that the same trend also holds for the $-20 \mathrm{~dB}$ spectral width. As far as the ripple of the spectrum in the central part of the pulse is concerned, our experimental results of Fig. 9(c) follow the expected trends, and clearly demonstrate the significance of the wave-breaking borderline in separating two welldistinct pulse propagation regions.

\section{Impact of losses and gain}

Throughout our previous analysis, we neglected the impact of losses and gain by considering $\delta=$ 0. In this section, we intend to lift that restriction and theoretically describe the effects on pulse dynamics of loss or amplification. Moreover, we shall propose an extension of Eq. (11) by taking into account a non-zero value of $\delta$.

We may follow a reasoning similar to that introduced by Anderson et al [26], which in the lossless case led us to the conclusions that permitted us to arrive at Eq. (11). In the presence of $\delta$, the nonlinear chirp which is induced by self-phase modulation may be estimated as [20, 38]:

$$
C_{n l}^{\prime}(\xi, \tau)=-\frac{1-\exp (-\xi \delta)}{\delta} \frac{\partial}{\partial \tau}\left(u^{2}(0, \tau)\right)
$$


Therefore the new limit distance for WB, say $\xi_{w b}$, is obtained from

$$
\xi_{w b} \frac{1-\exp \left(-\xi_{w b}^{\prime} \delta\right)}{\delta}=\frac{1}{4 \exp (-3 / 2) N^{2}}
$$

By introducing the usual effective length $\xi_{w b e f f}^{\prime}=\left(1-\exp \left(-\xi_{w b}^{\prime} \delta\right)\right) / \delta$ [20], Eq. (20) can be rewritten as :

$$
\xi_{w b}^{\prime} \xi_{w b e f f}^{\prime}=\frac{1}{4 \exp (-3 / 2) N^{2}}
$$

It should be pointed out that Eq. (21) is not simply the direct transposition of Eq. (10), as it could be obtained by replacing $\xi_{w b}$ by $\xi_{\text {wbeff }}$ : as a matter of fact, Eq. (21) involves the product of the distinct quantities $\xi_{w b}^{\prime} \xi_{w b e f f}^{\prime}$.

We have checked in Fig. 10 the validity of our proposed formula (20). In order to illustrate our approach, we have considered the linear normalized losses $\delta=200 \mathrm{~dB}$, and gain $\delta=-200 \mathrm{~dB}$. Let us recall that these gain/loss values are normalized relatively to the dispersion length. Such values can be typical for picosecond pulses propagating in rare-earth doped fibers and photonic crystal fibers, respectively. We have plotted on Figs. 10(a) and 10(b) the resulting evolution of the temporal slope of the pulse, for the case of an initial Gaussian pulse shape, and for either a lossy or active fiber, respectively. The predictions given by the analytical formula for the lossless case (Eq. (9)) are also shown in Fig. 10 as a solid white curve. The results of Fig. 10 clearly prove that our formula (20) provides a good description of the observed WB borderline in the presence of either distributed loss or gain. In the lossy case, Fig. 10(a) shows that the WB 
limit is shifted towards longer fiber distances. The contrary occurs for an active fiber, namely Fig. 10(b) shows that the WB boundary is shifted to shorter propagation distances.

It is also of great interest to compare the spectral profiles of the pulses as they emerge from the fiber at precisely the WB distance as it is predicted by Eq. (20). The black curves in Figs. 11(a) and (b) were obtained for an input Gaussian pulse with $N=40$, after propagation until $\xi_{w b}$ in a lossy or amplifying fiber, respectively. The comparison of Figs. 11(a) and (b) shows that amplified pulses develop a broader spectrum than linearly attenuated pulses. Another point which is also apparent from the inspection of Fig. 11 is that, at distances past the WB borderline of Eq. (20) (e.g., grey curves obtained at a distance $\xi=1.5 \xi_{w b}$ ), the spectral evolutions in the lossy and in the active case are rather different. Indeed, Fig. 11 shows that in the case of a lossy fiber the wings of the pulse spectra remain rather moderate at any distance. This can be explained by the relatively high attenuation value: upon the propagation, loss progressively decreases the pulse peak power: as a consequence, whenever intra-pulse overlap among different frequencies occurs, the FWM efficiency has dropped to dramatically low values. Consequently, only moderate wings development occurs, and the oscillations that are observed in the temporal profile are mainly due to the simple linear overlap of pulse sections with different instantaneous frequencies [39]. Fig. 11(a) shows another consequence of the presence of linear loss, namely the central portion of the pulse spectrum remains virtually undepleted. As a result the pulse spectrum exhibits the characteristic peaks at its extreme frequencies.

Quite to the contrary, in the case of an amplifying fiber the nonlinear interaction during the intra-pulse overlap of different frequencies is enhanced by gain. As a consequence, the wings that are the signature of WB develop more strongly than in the lossless case, while the central portion of the pulse spectrum gets depleted and smoothens more quickly. Those qualitative 
remarks help us to better understand the different experimental results that were recently obtained in a normal, varying dispersion fiber [16, 40]. Indeed, those experiences have demonstrated that the shape of the output pulse spectrum shape was remarkably different, depending upon the relative direction of the pulse propagation and the dispersion decrease. To this end, let us recall that a dispersion variation with distance can be formally linked to the presence of either gain or loss, depending upon the relative direction of dispersion decrease and pulse propagation. In fact a dispersion decreasing fiber leads to an equivalent gain, whereas a dispersion increasing fiber can be assimilated to a lossy fiber. In carrying out the comparison between the present results and earlier experiments, we should be careful however, that in this paper and in Fig.11 we compare the evolution in lossy and in active fibers of pulses with identical values of $\mathrm{N}$. On the other hand, when injecting pulses with the same input peak-power at the two ends of a varying dispersion fiber, clearly one obtains different values of $\mathrm{N}$ to start with.

Let us finally focus our attention on pulse evolution in amplifying fibers. This topic has attracted much interest over the past few years, mainly due to the experimental discovery of the formation of self-similar pulses [19] in media such as erbium or ytterbium doped fibers, Raman amplifiers [35] or dispersion decreasing fibers [16]. Indeed, it has been shown that any pulse, whatever its initial shape, asymptotically converges towards a parabolic intensity profile combined with a linear chirp, and then maintains this shape unchanged while undergoing a selfsimilar evolution.

In Fig. 12(a) we plotted the evolution with fiber length of the temporal width of an initial Gaussian pulse, for various $N$ values and for a gain $\delta=-200 \mathrm{~dB}$. As it can be seen, in a first stage 
of propagation there is hardly any change of the pulse temporal width. Next, after a certain distance of propagation which decreases as the initial $N$ grows larger, the pulse temporal broadening increases according to a self-similar evolution law which reads as $[19,38]$ :

$$
\frac{\tau_{-3}(\xi, N)}{\tau_{-3}(\xi=0)}=\frac{3}{\sqrt{2 \ln (2)}}\left(\frac{\sqrt{\pi}}{2} \frac{N^{2}}{\delta^{2}}\right)^{\frac{1}{3}} \exp \left(\frac{\delta \xi}{3}\right)
$$

In this context, it is of interest to display on Fig. 12(a), as we have done on the various curves associated with each value of $N$ by means of a full dot, the WB distance as it is predicted by Eq. (20). This permits us to reveal that the onset of WB appears as the turning point in the evolution of an initial Gaussian pulse towards an asymptotic parabolic pulse.

In order to attain a more precise knowledge of the temporal evolution of the pulse shape, we have computed the misfit parameter between the propagated pulse and its parabolic fit $p$ : such misfit may be defined as follows [31] :

$$
M^{2}=\int\left[|u|^{2}-|p|^{2}\right]^{2} d \tau / \int|u|^{4} d \tau
$$

The corresponding results are plotted in Fig. 12(b), along with the WB distance as a dotted white curve. In Fig. 12(b) we may clearly see that an initial Gaussian pulse approaches a parabolic shape exactly at the limit distance for WB that is predicted by Eq. (20), in agreement with previously demonstrated results and observations carried out in passive fibers [31]. However Fig. 12(b) also shows that, for large values of $N$, the parabolic shape which is attained at $\xi_{w b}$ cannot be maintained in further propagation, and the pulse undergoes wave-breaking effects, alongside 
with all their classical manifestations (i.e., the development of wings in the spectrum, rapid oscillations of the temporal intensity profile). Nevertheless, Fig. 12(b) also reveals that soon thereafter the pulse stably converges towards a self-similar solution of the propagation equation, that is the pulse definitively acquires a parabolic intensity profile, as shown by corresponding low values of the $M$ factor. A similar evolution could already be seen in Ref. [38] (Fig . 1(b)), even though at that time no clear interpretation of this behaviour was provided.

In summary, the results of the present section provide what we believe is a significant insight for a better comprehension of the evolution of arbitrary optical pulses towards a parabolic state, a topic which has generated widespread concern in the recent literature [31, 41-43]. From the results of Fig.12, we may conclude that even if the temporal width of a propagated pulse evolves according with the self-similar evolution of a parabolic pulse past the point where WB is generated, the full shape of this pulse may still oscillate around its asymptotic state. Nevertheless, the presence of WB remains an important turning point in the process of attraction of the initial pulse towards the parabolic shape. Indeed, quite surprisingly we have revealed that although an exactly parabolic pulse is exempt from the WB effect [36], yet an arbitrary input pulse may still experience the WB effect along its evolution towards the asymptotic, stable selfsimilar parabolic state.

\section{Conclusion}

In this article we have carried out a detailed temporal and spectral analysis of pulse propagation in a normally dispersive optical fiber in the presence of Kerr nonlinearity. We have outlined the crucial role that is played by the wave-breaking effect in determining the pulse reshaping. 
Moreover, we have interpreted the action of wave-breaking in terms of fundamental physical intra-pulse interactions. We have revealed that the threshold for wave-breaking separates qualitatively different regimes of pulse reshaping in both temporal and frequency domains, which is of great relevance for different practical applications. We have compared and validated our numerical and analytical predictions with direct systematic experimental studies, confirming that wave-breaking represents a key ingredient in the formation of highly coherent continuum pulses. Based on simple physical arguments, we have proposed analytical guidelines for predicting the evolution of the main characteristics of the optical pulses. Finally, we have extended our concepts to the case of pulse propagation in a lossy medium, as well as in fiber amplifiers. In the last case we have pointed out that, quite surprisingly, a pulse may still undergo the wave-breaking effect upon its attraction towards a self-similar parabolic pulse.

\section{Acknowledgement}

This research was supported by the Agence Nationale de la Recherche (SUPERCODE project, project BLAN-0401) and by the Conseil Régional de Bourgogne. We would like to acknowledge Guy Millot and Dave Richardson for fruitful discussions. 


\section{References}

1. J. M. Dudley, G. Genty, and S. Coen, "Supercontinuum generation in photonic crystal fiber," Rev. Modern Physics 78, 1135-1184 (2006).

2. I. Hartl, X. D. Li, C. Chudoba, R. K. Ghanta, T. H. Ko, J. G. Fujimoto, J. K. Ranka, and R. S. Windeler, "Ultrahigh-resolution optical coherence tomography using continuum generation in an air-silica microstructure optical fiber," Opt. Lett. 26, 608-610 (2001).

3. S. A. Diddams, D. J. Jones, J. Ye, T. Cundiff, J. L. Hall, J. K. Ranka, R. S. Windeler, R. Holzwarth, T. Udem, and T. W. Hanch, "Direct link between microwave and optical frequencies with a $300 \mathrm{THz}$ femtosecond laser comb," Phys. Rev. Lett. 84, 5102-5105 (2000).

4. L. Boivin and B. C. Collings, "Spectrum slicing of coherent sources in optical communications," Opt. Fiber. Technol. 7, 1-20 (2001).

5. F. Parmigiani, C. Finot, K. Mukasa, M. Ibsen, M. A. F. Roelens, P. Petropoulos, and D. J. Richardson, "Ultra-flat SPM-broadened spectra in a highly nonlinear fiber using parabolic pulses formed in a fiber Bragg grating," Opt. Express 14, 7617-7622 (2006).

6. Z. Yousoff, P. Petropoulos, F. Furusawa, T. M. Monro, and D. J. Richardson, "A 36channel x 10-GHz spectrally sliced pulse source based on supercontinuum generation in normally dispersive highly nonlinear holey fiber," IEEE Photon. Technol. Lett. 15, 16891691 (2003).

7. T. Morioka, K. Mori, and M. Saruwatari, "More than 100-wavelength-channel picosecond optical pulse generation from single laser source using supercontinuum in optical fibres," Electron. Lett. 29, 862-864 (1993).

8. Y. Takushima and K. Kikuchi, "10-GHz, over 20-channel multiwavelength pulse source by slicing super-continuum spectrum generated in normal-dispersion fiber," IEEE Photon. Technol. Lett. 11, 322-424 (1999).

9. M. Nakazawa, K. Tamura, H. Kubota, and E. Yoshida, "Coherence degradation in the process of supercontinuum generation in an optical fiber," Opt. Fiber. Technol. 4, 215223 (1998). 
10. N. Nishizawa and J. Takayanagi, "Octave spanning high-quality supercontinuum generation in all-fiber system," J. Opt. Soc. Am. B 24, 1786-1792 (2006).

11. P. V. Mamyshev, "All-optical data regeneration based on self-phase modulation effect," in European Conference on Optical Communication, ECOC'98, 1998), 475-476.

12. L. Provost, C. Finot, K. Mukasa, P. Petropoulos, and D. J. Richardson, "Design scaling rules for 2R-Optical Self-Phase Modulation-based regenerators 2R regeneration," Opt. Express 15, 5100-5113 (2007).

13. L. Fu, V. G. Ta'eed, E. C. Mägi, I. C. M. Littler, M. Pelusi, M. R. E. Lamont, A. Fuerbach, H. C. Nguyen, D. I. Yeom, and B. J. Eggleton, "Highly nonlinear chalcogenide fibres for all-optical signal processing," Optical and Quantum Electronics.

14. W. J. Tomlinson, R. H. Stolen, and C. V. Shank, "Compression of optical pulses chirped by self-phase modulation in fibers," J. Opt. Soc. Amer. B 1, 139-149 (1984).

15. D. Grischkowsky and A. C. Balant, "Optical pulse compression based on enhanced frequency chirping," Appl. Phys. Lett. 41, 1-3 (1982).

16. C. Finot, B. Barviau, G. Millot, A. Guryanov, A. Sysoliatin, and S. Wabnitz, "Parabolic pulse generation with active or passive dispersion decreasing optical fibers," Opt. Express 15, 15824-15835 (2007).

17. H. Nakatsuka, D. Grischkowsky, and A. C. Balant, "Nonlinear picosecond-pulse propagation through optical fibers with positive group velocity dispersion," Phys. Rev. Lett. 47, 910-913 (1981).

18. W. J. Tomlinson, R. H. Stolen, and A. M. Johnson, "Optical wave-breaking of pulses in nonlinear optical fibers," Opt. Lett. 10, 457-459 (1985).

19. J. M. Dudley, C. Finot, G. Millot, and D. J. Richardson, "Self-similarity in ultrafast nonlinear optics," Nat. Phys. 3, 597-603 (2007).

20. G. P. Agrawal, Nonlinear Fiber Optics, Third Edition (Academic Press, San Francisco, CA, 2001).

21. E. Bourkoff, W. Zhao, R. I. Joseph, and D. N. Christodoulides, "Evolution of femtosecond pulses in single-mode fibers having higher-order nonlinearity and dispersion," Opt. Lett. 12, 272-274 (1987). 
22. A. Ruehl, O. Prochnow, D. Wandt, D. Kracht, B. Burgoyne, N. Godbout, and S. Lacroix, "Dynamics of parabolic pulses in an ultrafast fiber laser," Opt. Lett. 31, 2734-2736 (2006).

23. C. Jirauschek, F. Ö. Ilday, and F. X. Kärtner, "A Semi-Analytic Theory of the SelfSimilar Laser Oscillator," in Non Linear Guided Waves and their Applications (NLGW), 2005),

24. B. Burgoyne, N. Godbout, and S. Lacroix, "Nonlinear pulse propagation in optical fibers using second order moments," Opt. Express 15, 10075-10090 (2007).

25. C.-K. Rosenberg, D. Anderson, M. Desaix, P. Johannisson, and M. Lisak, "Evolution of optical pulses towards wave breaking in highly nonlinear fibres," Opt. Commun. 273, 272-277 (2007).

26. D. Anderson, M. Desaix, M. Lisak, and M. L. Quiroga-Teixeiro, "Wave-breaking in nonlinear optical fibers," J. Opt. Soc. Am. B 9, 1358-1361 (1992).

27. J. P. Hamaide and P. Emplit, "Direct observation of optical wave breaking of picosecond pulses in nonlinear single-mode optical fibres," Electron. Lett. 24, 818-819 (1988).

28. S. Linden, H. Giessen, and J. Kruhl, "XFROG - A new method for amplitude and phase characterization of weak ultrashort pulses," Phys. Stat. Sol. 206, 119-124 (1998).

29. J. Azana, "Time-frequency (Wigner) analysis of linear and nonlinear pulse propagation in optical fibers," EURASIP Journal on Applied Signal Processing 10, 1554-1565 (2005).

30. R. H. Stolen and Q. Lin, "Self-phase modulation in silica optical fibers," Phys. Rev. A 17, 1448-1453 (1978).

31. C. Finot, L. Provost, P. Petropoulos, and D. J. Richardson, "Parabolic pulse generation through passive nonlinear pulse reshaping in a normally dispersive two segment fiber device," Opt. Express 15, 852-864 (2007).

32. J. E. Rothenberg, "Femtosecond optical shocks and wave-breaking in fiber propagation," J. Opt. Soc. Amer. B 6, 2392-2401 (1989).

33. J. E. Rothenberg, "Colliding visible picosecond pulses in optical fibers," Opt. Lett. 15, 443-445 (1990).

34. S. Taccheo and L. Boivin, "Investigation and design rules of supercontinuum sources for WDM applications," in Optical Fiber Communication, 2000), ThA1. 
35. C. Finot and G. Millot, "Synthesis of optical pulses by use of similaritons," Opt. Express 12, 5104-5109 (2004).

36. D. Anderson, M. Desaix, M. Karlson, M. Lisak, and M. L. Quiroga-Teixeiro, "Wavebreaking-free pulses in nonlinear optical fibers," J. Opt. Soc. Am. B 10, 1185-1190 (1993).

37. D. Schadt and B. Jaskorzynska, "Frequency chirp and spectra due to self-phase modulation and stimulated Raman scattering influenced by pulse walk-off in optical fibers," J. Opt. Soc. Am. B 4, 856-862 (1987).

38. C. Finot, F. Parmigiani, P. Petropoulos, and D. J. Richardson, "Parabolic pulse evolution in normally dispersive fiber amplifiers preceding the similariton formation regime," Opt. Express 14, 3161-3170 (2006).

39. H. E. Lassen, F. Mengel, B. Tromborg, N. C. Albertsen, and P. L. Christiansen, "Evolution of chirped pulses in nonlinear single-mode fibers," Opt. Lett. 10, 34-36 (1985).

40. A. Plocky, A. A. Sysoliatin, A. I. Latkin, V. F. Khopin, P. Harper, J. Harrison, and S. K. Turitsyn, "Experiments on the generation of parabolic pulses in waveguides with lengthvarying normal chromatic dispersion," JETP Letters 85, 319-322 (2007).

41. V. I. Kruglov and J. D. Harvey, "Asymptotically exact parabolic solutions of the generalized nonlinear Schrödinger equation with varying parameters," J. Opt. Soc. Amer. B 23, 2541-2550 (2006).

42. S. Wabnitz, "Analytical Dynamics of parabolic pulses in nonlinear optical fiber amplifiers," IEEE Photon. Technol. Lett. 19, 507-509 (2007).

43. Y. Ozeki, Y. Takushima, K. Taira, and K. Kikuchi, "Clean similariton generation from an initial pulse optimized by the backward propagation method," in Conference on Lasers and Electro-Optics (CLEO US), (OSA Trends in Optics and Photonics Series, 2004), CTuBB51113-51114. 


\section{Figures captions}

1. Characteristic parameters computed for pulse analysis (a) in the temporal domain (b) in the spectral domain.

2. Mixed spectral-temporal representation of the optical pulse at various propagation lengths.

3. Computed evolution of the temporal properties of initial Gaussian pulse vs. its propagation distance $\xi$ and $N$ number: (a) Broadening factor of the temporal FWHM; (b) Peak-power decrease factor. The solid white curve is the analytical WB boundary of Eq. (10).

4. Computed evolution of various pulse properties: (a) slope $S$; (b) parameter $F$; (c) linear slope of the temporal chirp coefficient. The analytical prediction of WB boundary in Eq. (10) is plotted as solid white line.

5. Computed evolution of the pulse spectral properties: (a) -3dB spectral broadening factor; (b) maximum spectral broadening for Gaussian and sech initial pulses. Numerical results are plotted with black diamonds (Gaussian pulses) or with grey circles (sech pulses). The solid lines are the theoretical results from Eq. (16) (black line, for Gaussian initial pulses) or Eq. (18) (grey line, for initial sech pulses); (c) Evolution of the $-20 \mathrm{~dB}$ spectral width; (d) Ratio between the $-3 \mathrm{~dB}$ and $20 \mathrm{~dB}$ spectral widths. 
6. Computed evolution of the pulse spectral properties: (a) evolution of the fraction of pulse energy contained in the central part of its spectrum ( $3 \mathrm{~dB}$ bandwidth); (b) evolution of the pulse spectral ripple. The solid white curve indicates the analytical WB boundary of Eq. (10).

7. Computed evolution of the energy fraction stored in the central region of the pulse spectrum, for a sech pulse (a) or for a parabolic pulse (b); The prediction of Eq. (17) is shown with a black solid line.

8. Experimental set-up.

9. Experimental maps for: (a) the evolution of the $-3 \mathrm{~dB}$ spectral width; (b) the $-20 \mathrm{~dB}$ spectral width; (c) the spectral ripple. Crosses indicate discarded data points where Raman scattering was significant. The analytical predictions from Eq. (17) are plotted as a white solid curve.

10. Map showing the computed evolution of temporal slope $S$ of the pulse for: (a) a lossy fiber with $\delta=200 \mathrm{~dB}$; (b) or an amplifying fiber with $\delta=-200 \mathrm{~dB}$. Theoretical borders given by Eq. (20) for losses and gain are plotted using white dashed or dashed-dotted lines, respectively. The solid white line represents the lossless case.

11. Computed output spectra of an initially Gaussian pulse, after propagation in normally dispersive fiber until the WB distance of Eq. (20) (black curves) or at $1.5 \xi_{w b}$ (grey curves) for $N$ $=40$ : (a) case of a lossy fiber (b) case of an amplifying fiber. 
12. (a) Computed evolution of the FWHM temporal pulse broadening for various $N$ values ( $N=$ 5, 15, 25 and 35, solid lines, decreasing grey levels from black to light grey). Computed evolutions are compared with the corresponding asymptotic evolutions as described by Eq. (22) (dashed lines). Filled round points indicate the WB distance as it is predicted by Eq. (20); (b) computed evolution of the misfit parameter $M$. 

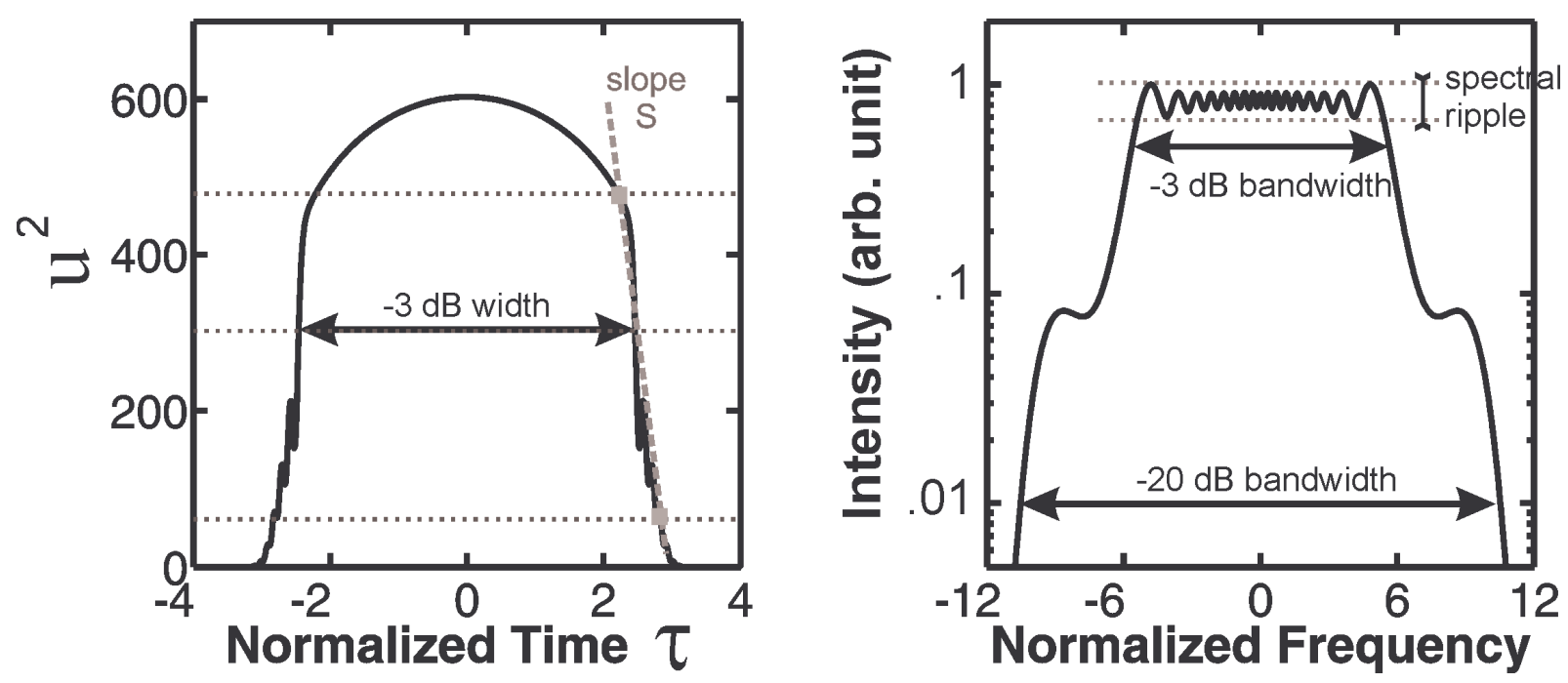

Figure 1 


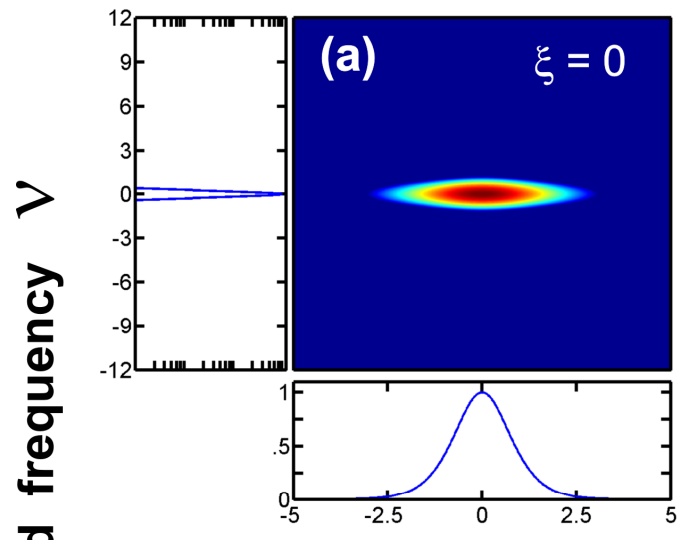

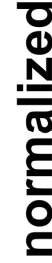

हํํำ

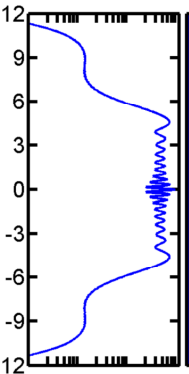

(c)

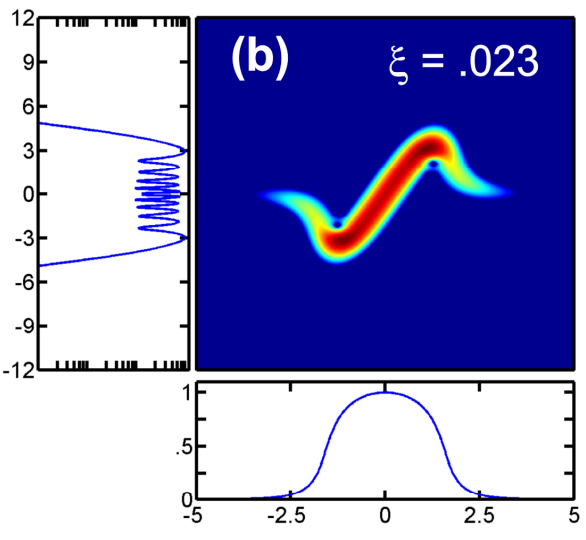

(d)

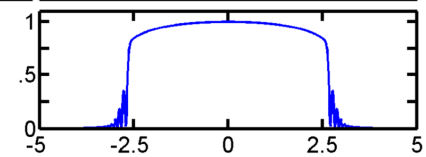

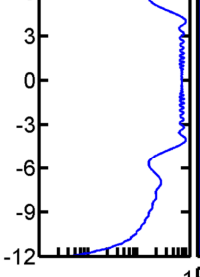

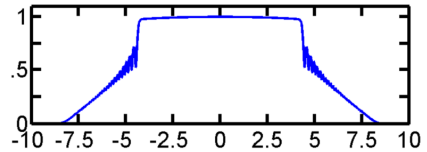

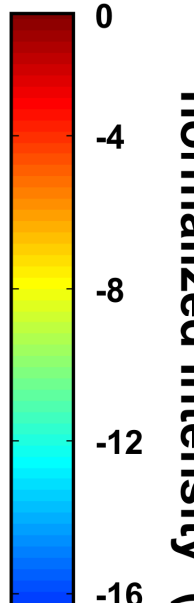

$-16$

产

normalized time $\tau$

Figure 2 


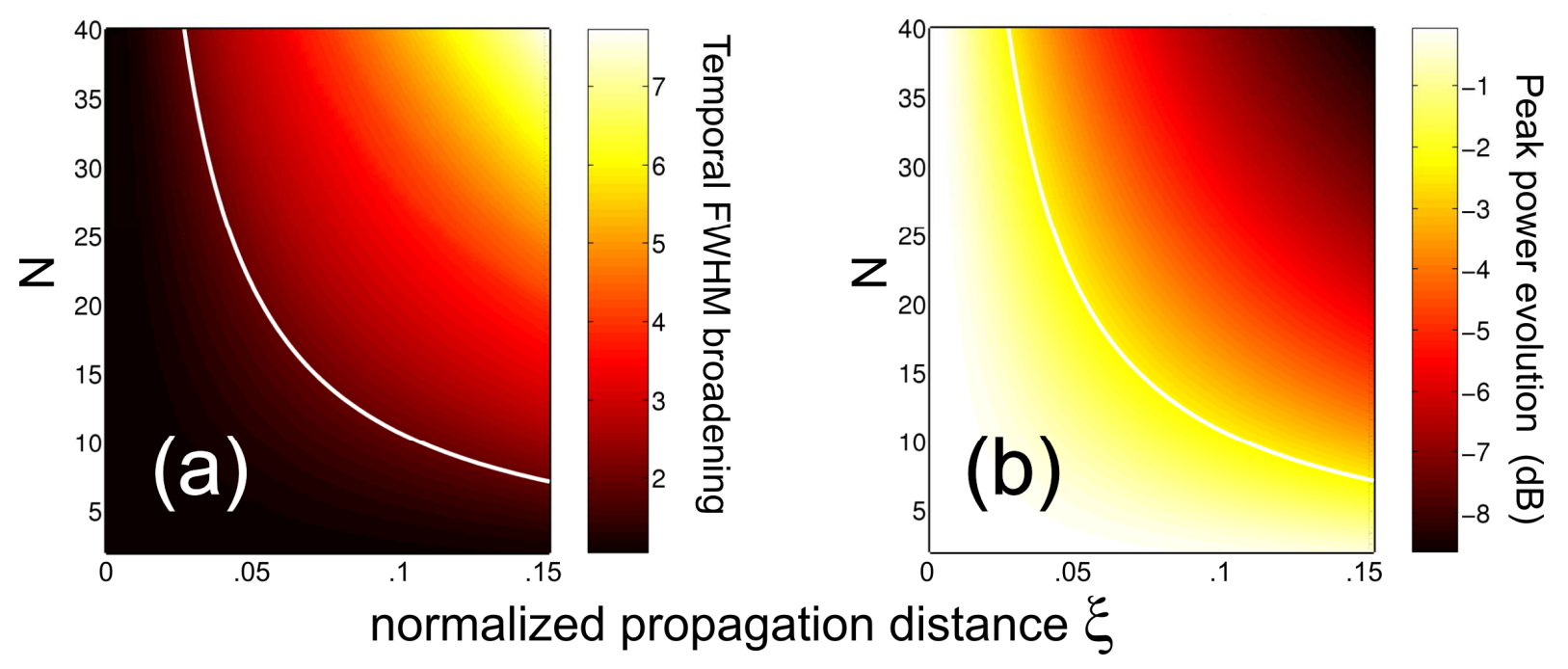

Figure 3 

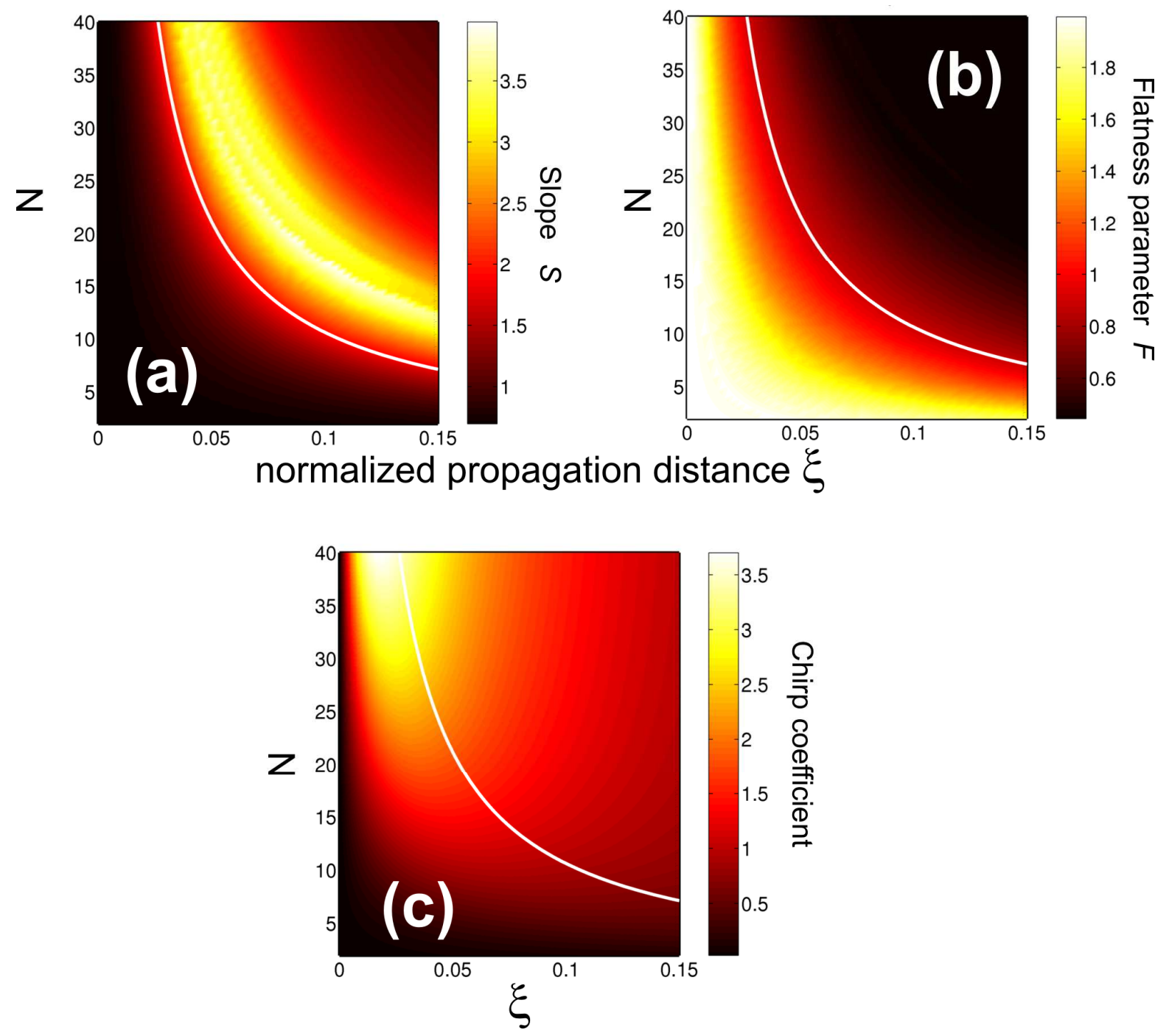

Figure 4 

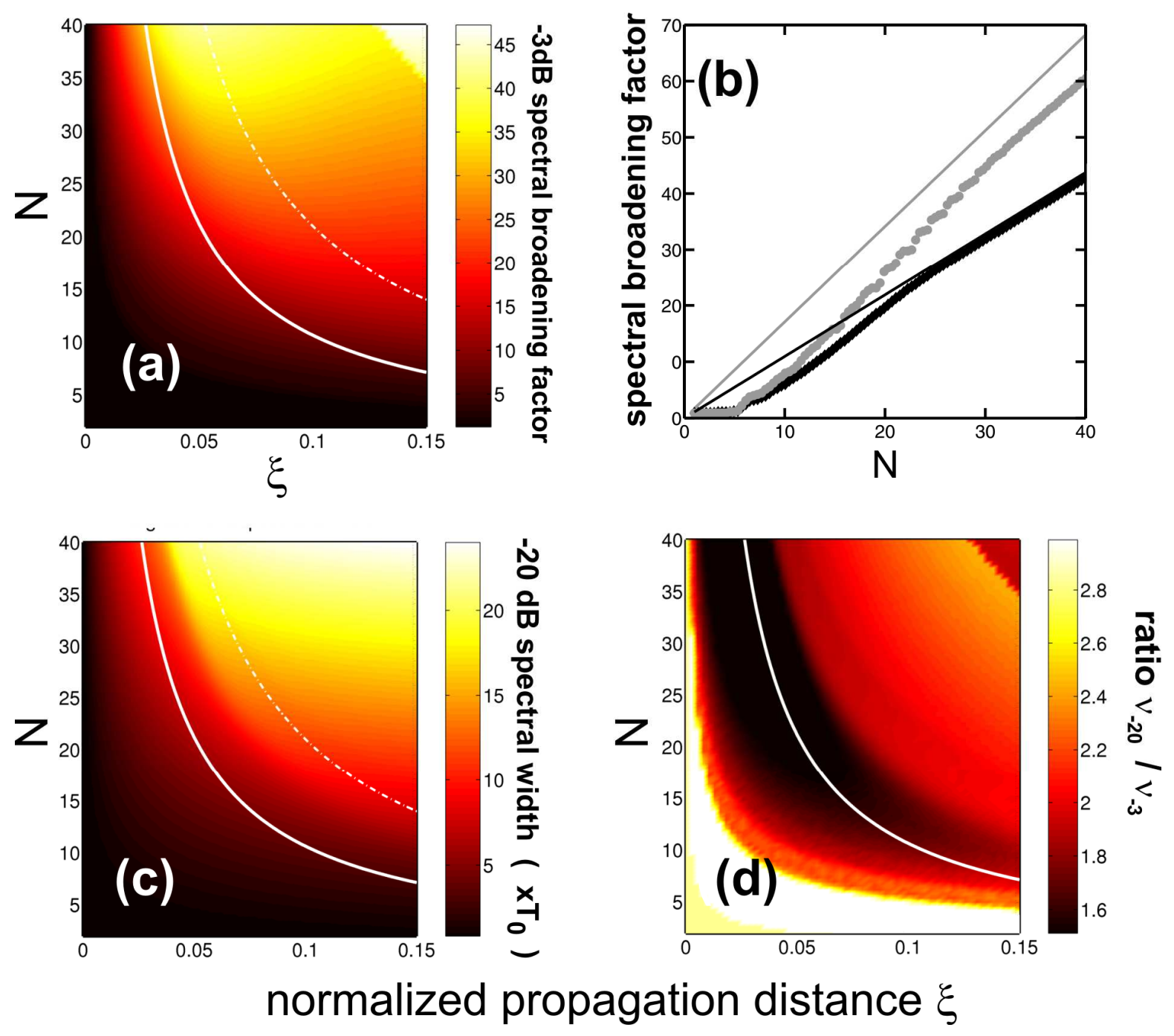

Figure 5 


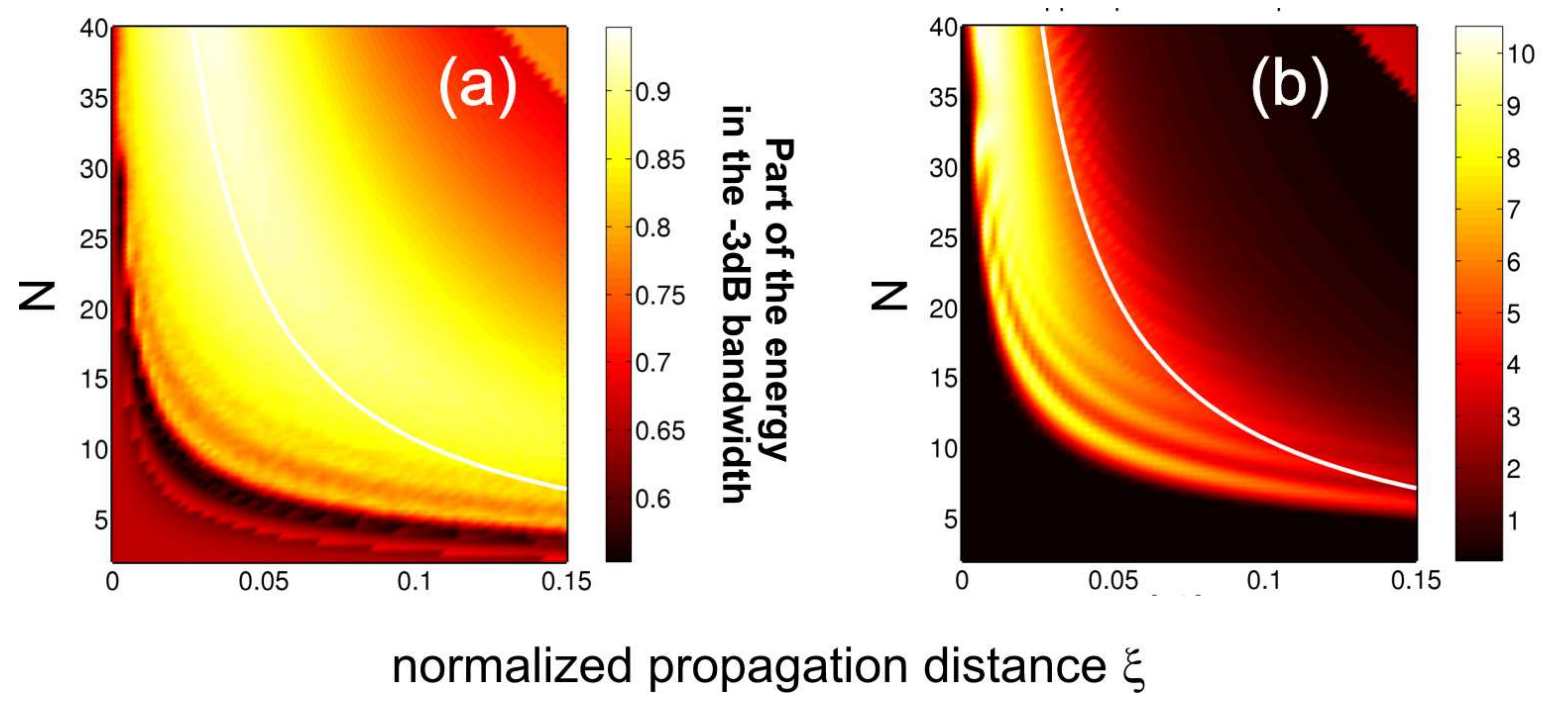

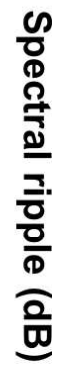

Figure 6 


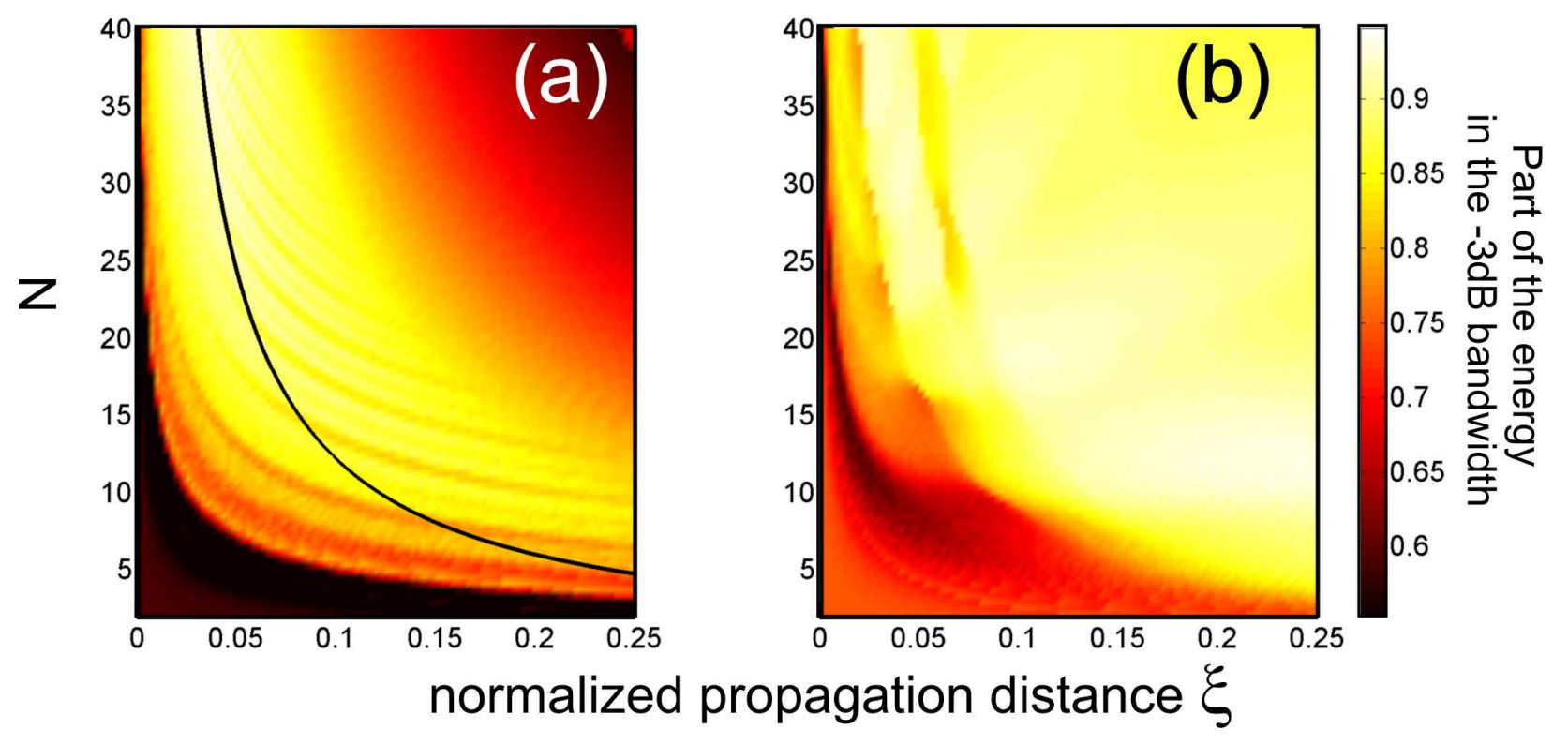

Figure 7 


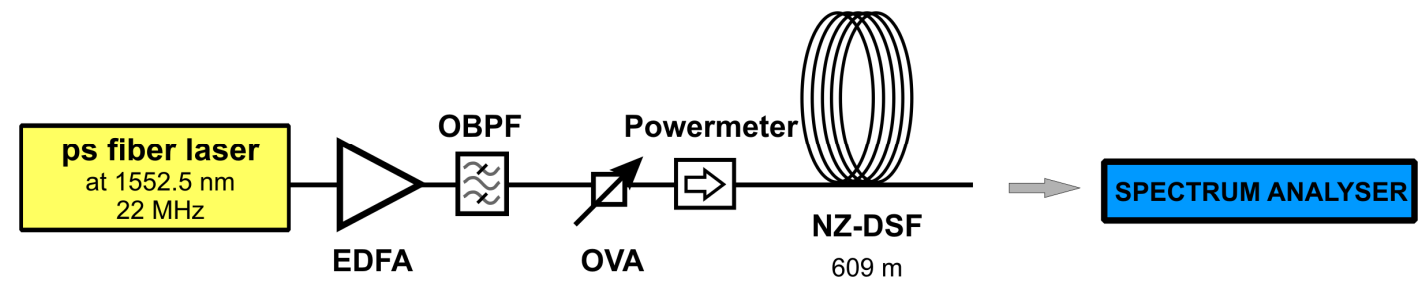

Figure 8 

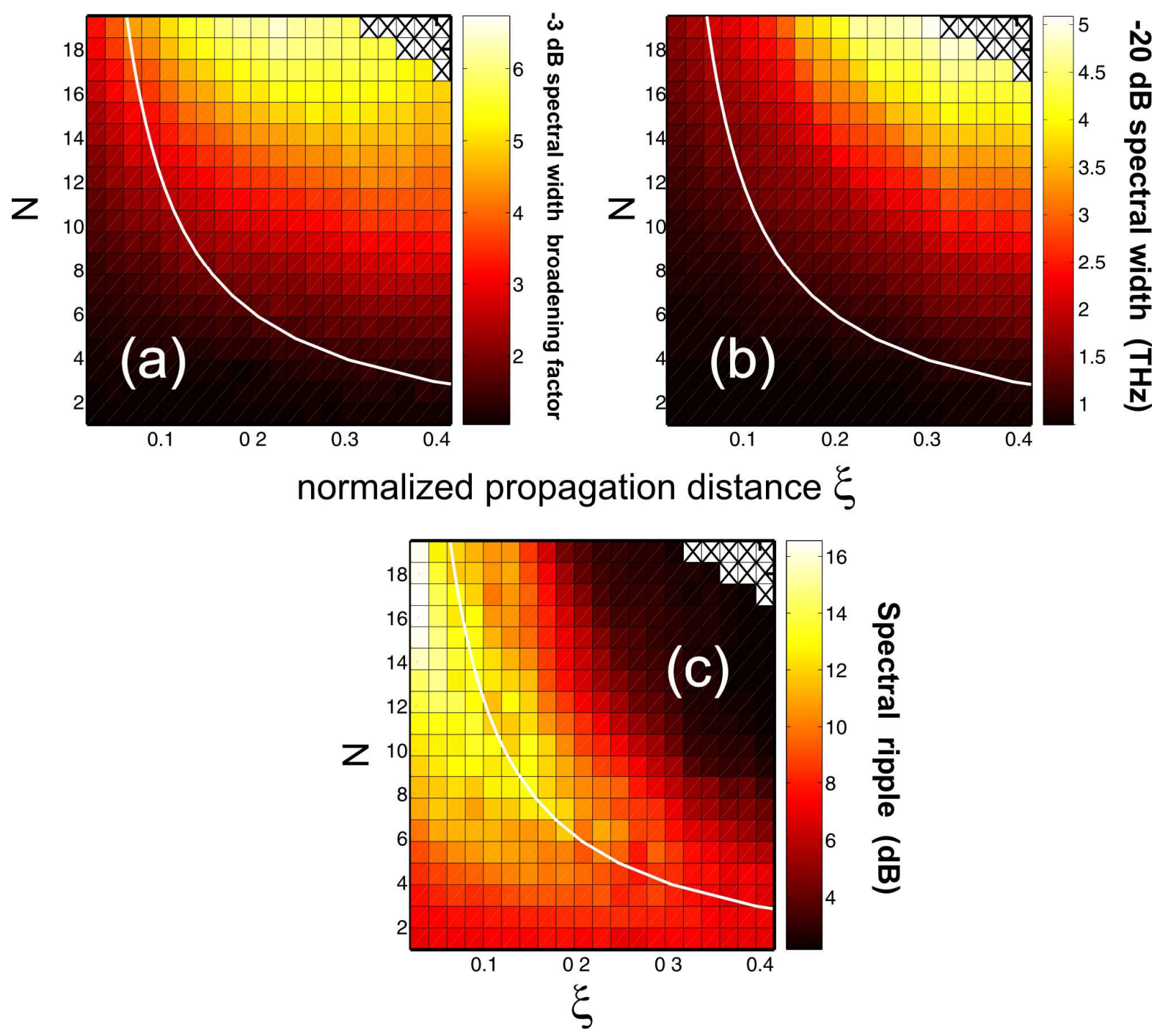

Figure 9 


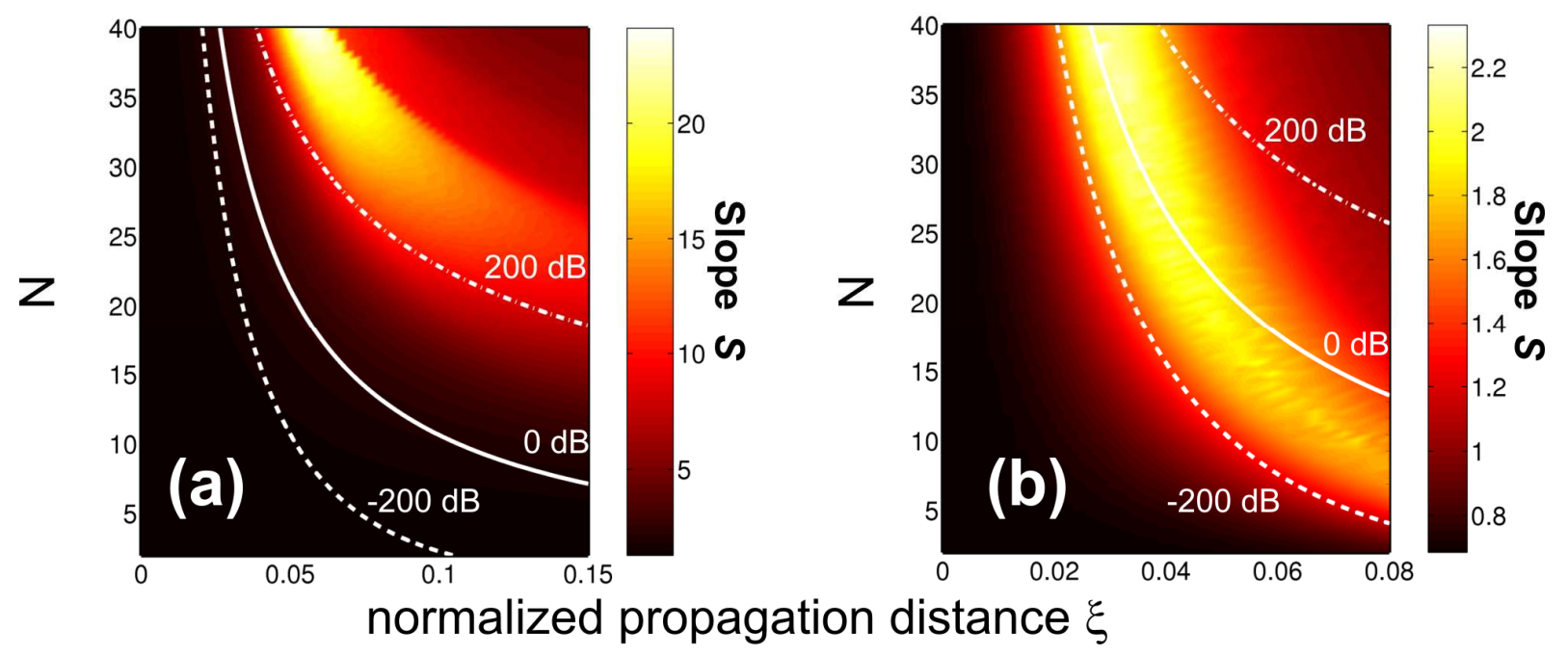

Figure 10 


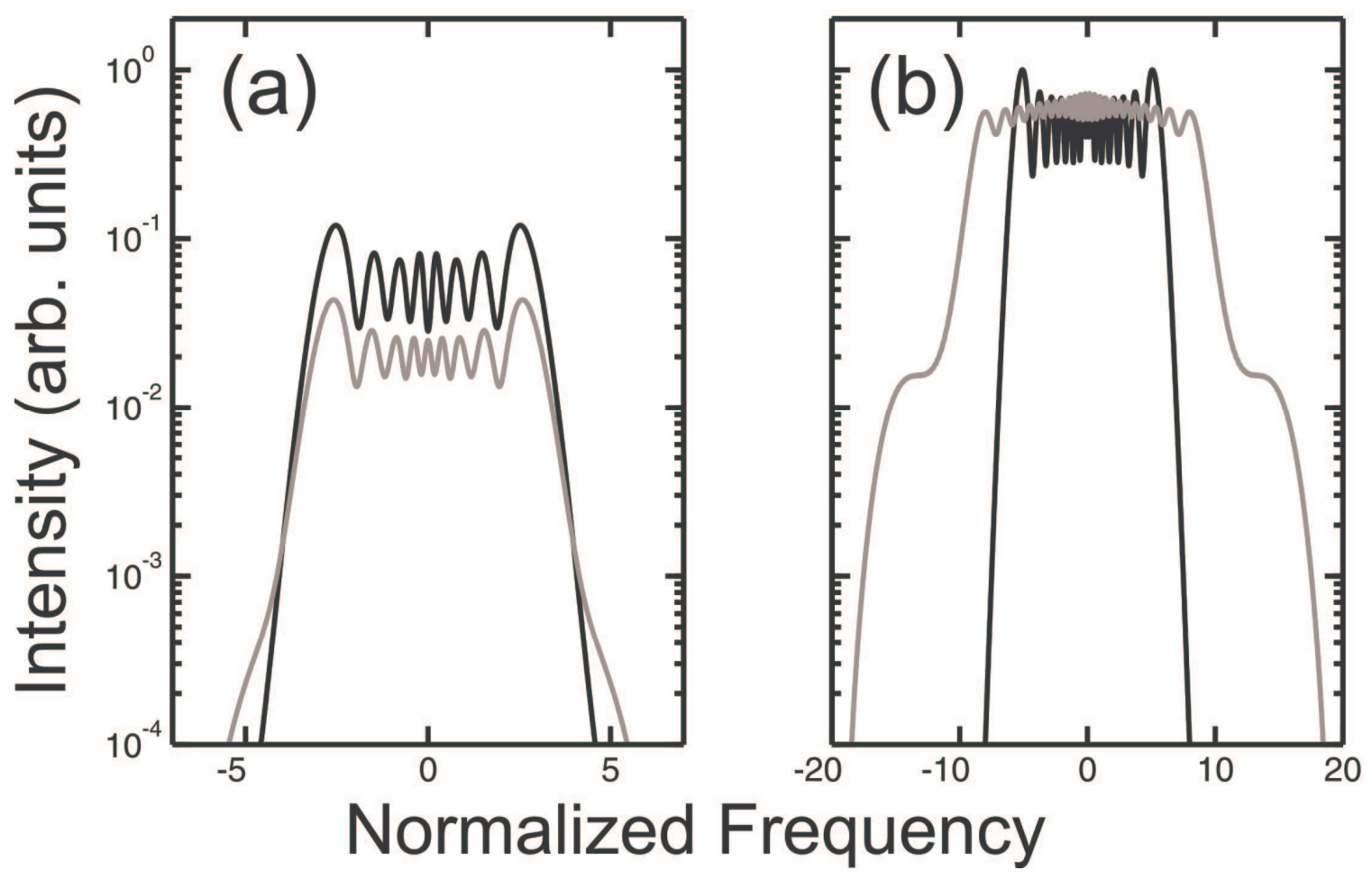

Figure 11 


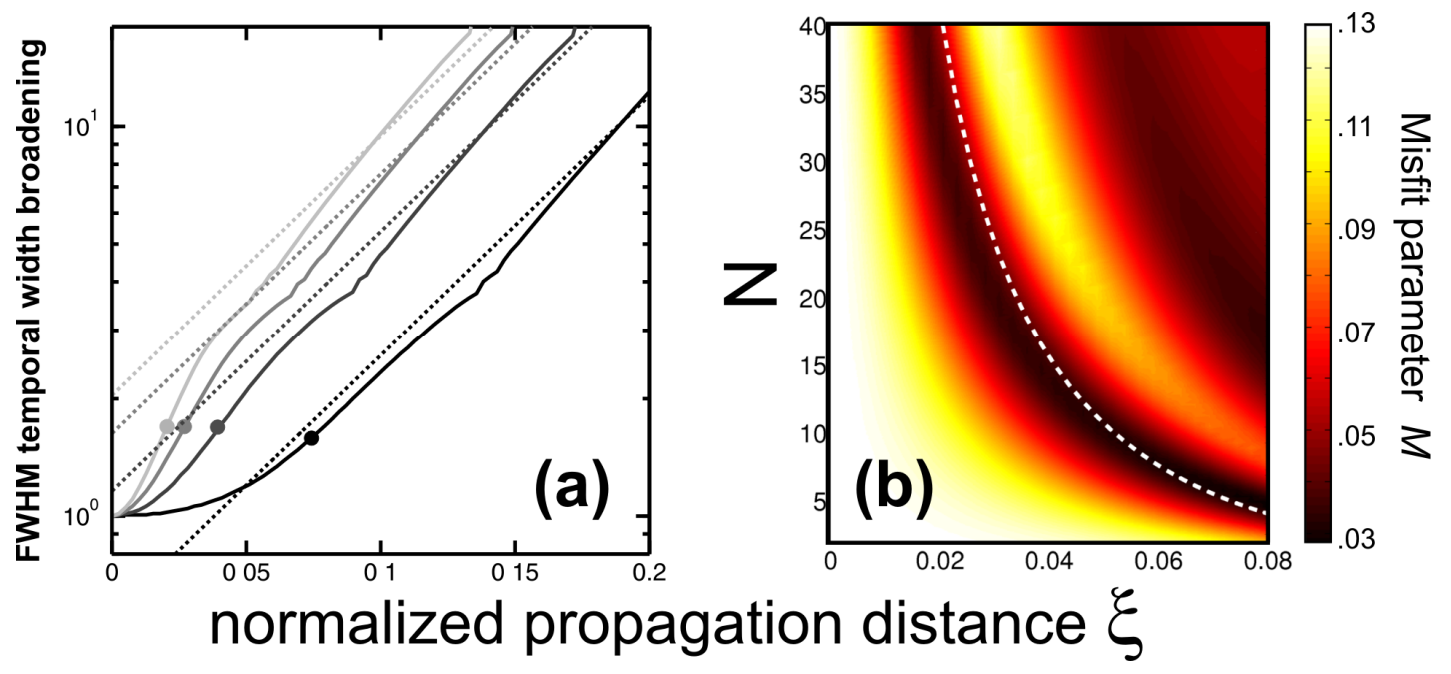

Figure 12 